Supporting Information for:

\title{
Solution Structure of the K50 Class Homeodomain PITX2 Bound to DNA and Implications for Mutations that Cause Rieger Syndrome
}

Beth A. Chaney, Kimber Clark-Baldwin, Vrushank Dave, Jun Ma, Mark Rance

Department of Molecular Genetics, Biochemistry, and Microbiology, University of Cincinnati, College of Medicine, 231 Albert Sabin Way. Medical Sciences Building, Cincinnati OH 45267-0524

\begin{tabular}{|c|c|c|c|c|c|c|c|c|c|c|c|c|c|c|c|c|c|c|c|c|c|c|c|c|c|c|c|c|c|c|}
\hline & & & & & & & & & & 10 & & & & & & & & & & 20 & & & & & & & & & & 30 \\
\hline Pitx2 & $\mathrm{Q}$ & $\mathrm{R}$ & $\mathrm{R}$ & $\mathrm{Q}$ & $\mathrm{R}$ & $\mathrm{T}$ & $\mathrm{H}$ & $F$ & $\mathrm{~T}$ & $S$ & $\mathrm{Q}$ & $\mathrm{Q}$ & $\mathrm{L}$ & Q & $\mathrm{Q}$ & $\mathrm{L}$ & $E$ & A & $\mathrm{T}$ & $\bar{F}$ & $\mathrm{Q}$ & $\mathrm{R}$ & $\mathrm{N}$ & $\mathrm{R}$ & $\mathrm{Y}$ & $\mathrm{P}$ & $\mathrm{D}$ & $\mathrm{M}$ & $\mathrm{S}$ & $\mathrm{T}$ \\
\hline Bcd & $\mathrm{P}$ & $\mathrm{R}$ & $\mathrm{R}$ & $\mathrm{T}$ & $\mathrm{R}$ & $\mathrm{T}$ & $\mathrm{T}$ & $F$ & $\mathrm{~T}$ & S & $S$ & $\mathrm{Q}$ & I & A & $E$ & $\mathrm{~L}$ & $\mathrm{E}$ & Q & $\mathrm{H}$ & $F$ & $\mathrm{~L}$ & $\mathrm{Q}$ & $\mathrm{G}$ & $\mathrm{R}$ & $\mathrm{Y}$ & $\mathrm{L}$ & $\mathrm{T}$ & $\mathrm{A}$ & $\mathrm{P}$ & $\mathrm{R}$ \\
\hline Antp & $\mathrm{R}$ & $\mathrm{K}$ & $\mathrm{R}$ & G & $\mathrm{R}$ & $\mathrm{Q}$ & $\mathrm{T}$ & $\mathrm{Y}$ & $\mathrm{T}$ & $\mathrm{R}$ & $\mathrm{Y}$ & $\mathrm{Q}$ & $\mathrm{T}$ & L & $E$ & $\mathrm{~L}$ & $\mathrm{E}$ & K & $\mathrm{E}$ & $F$ & $\mathrm{H}$ & $\mathrm{F}$ & $\mathrm{N}$ & $\mathrm{R}$ & $\mathrm{Y}$ & $\mathrm{L}$ & $\mathrm{T}$ & $\mathrm{R}$ & $\mathrm{R}$ & $\mathrm{R}$ \\
\hline En & $\mathrm{E}$ & $\mathrm{K}$ & $\mathrm{R}$ & $\mathrm{P}$ & $\mathrm{R}$ & $\mathrm{T}$ & A & $F$ & $S$ & $S$ & $\mathrm{E}$ & $\mathrm{Q}$ & $\mathrm{L}$ & A & $\mathrm{R}$ & $\mathrm{L}$ & $\mathrm{K}$ & $\mathrm{R}$ & $E$ & $F$ & $\mathrm{~N}$ & $E$ & $\mathrm{~N}$ & $\mathrm{R}$ & $\mathrm{Y}$ & $\mathrm{L}$ & $\mathrm{T}$ & $\mathrm{E}$ & $\mathrm{R}$ & $\mathrm{R}$ \\
\hline Ftz & $\mathrm{S}$ & $\mathrm{K}$ & $\mathrm{R}$ & $\mathrm{T}$ & $\mathrm{R}$ & $\mathrm{Q}$ & $\mathrm{T}$ & $\mathrm{Y}$ & $\mathrm{T}$ & $\mathrm{R}$ & $\mathrm{Y}$ & $\mathrm{Q}$ & $\mathrm{T}$ & $\mathrm{L}$ & $\mathrm{E}$ & $\mathrm{L}$ & $\mathrm{E}$ & K & $E$ & $\mathrm{~F}$ & $\mathrm{H}$ & $\mathrm{F}$ & $\mathrm{N}$ & $\mathrm{R}$ & $\mathrm{Y}$ & I & $\mathrm{T}$ & $\mathrm{R}$ & $\mathrm{R}$ & $\mathrm{R}$ \\
\hline Mat2 & $\mathrm{K}$ & $\mathrm{P}$ & $\mathrm{Y}$ & $\mathrm{R}$ & $G$ & $\mathrm{H}$ & $\mathrm{R}$ & $F$ & $\mathrm{~T}$ & $\mathrm{~K}$ & $E$ & $\mathrm{~N}$ & $\mathrm{~V}$ & $\mathrm{R}$ & I & $\mathrm{L}$ & $\mathrm{E}$ & $S$ & $\mathrm{~W}$ & $F$ & A & $\mathrm{K}$ & $\mathrm{N}^{*}$ & $\mathrm{P}$ & $\mathrm{Y}$ & $\mathrm{L}$ & $\mathrm{D}$ & $\mathrm{T}$ & $\mathrm{K}$ & $\mathrm{G}$ \\
\hline Vnd & $\mathrm{K}$ & $\mathrm{R}$ & $\mathrm{K}$ & $\mathrm{R}$ & $\mathrm{R}$ & $\mathrm{V}$ & $\mathrm{L}$ & $\mathrm{F}$ & $\mathrm{T}$ & $\mathrm{K}$ & $\mathrm{A}$ & $\mathrm{Q}$ & $\mathrm{T}$ & $\mathrm{Y}$ & $E$ & $\mathrm{~L}$ & $\mathrm{E}$ & $\mathrm{R}$ & $\mathrm{R}$ & $F$ & $\mathrm{R}$ & $\mathrm{Q}$ & Q & $\mathrm{R}$ & $\mathrm{V}$ & $\mathrm{L}$ & $S$ & A & $\mathrm{P}$ & $\mathrm{E}$ \\
\hline & & & & & & & & & & 40 & & & & & & & & & & 50 & & & & & & & & & & 60 \\
\hline Pitx2 & $\mathrm{R}$ & $E$ & $E$ & I & A & $\mathrm{V}$ & $\mathrm{W}$ & $\mathrm{T}$ & $\mathrm{N}$ & $\mathrm{L}$ & $\mathrm{T}$ & $E$ & $\bar{A}$ & $\mathrm{R}$ & $\mathrm{V}$ & $\mathrm{R}$ & $\mathrm{V}$ & $\mathrm{W}$ & $F$ & $\mathrm{~K}$ & $\mathrm{~N}$ & $\mathrm{R}$ & $\mathrm{R}$ & A & $\mathrm{K}$ & $\mathrm{W}$ & $\mathrm{R}$ & $\mathrm{K}$ & $\mathrm{R}$ & $\bar{E}$ \\
\hline Bcd & $\mathrm{L}$ & $\mathrm{A}$ & $\mathrm{D}$ & $\mathrm{L}$ & $S$ & A & $\mathrm{K}$ & $\mathrm{L}$ & A & $\mathrm{L}$ & $\mathrm{G}$ & $\mathrm{T}$ & A & Q & $\mathrm{V}$ & $\bar{K}$ & I & $\mathrm{W}$ & $\mathrm{F}$ & $\mathrm{K}$ & $\mathrm{N}$ & $\mathrm{R}$ & $\mathrm{R}$ & $\mathrm{R}$ & $\mathrm{R}$ & $\mathrm{H}$ & $\mathrm{K}$ & I & $\mathrm{Q}$ & $S$ \\
\hline Antp & $\mathrm{R}$ & I & $E$ & I & A & $\mathrm{H}$ & A & $\mathrm{L}$ & $\mathrm{C}$ & $\mathrm{L}$ & $\mathrm{T}$ & $E$ & A & Q & I & $\bar{K}$ & I & $\mathrm{W}$ & $\mathrm{F}$ & $\mathrm{Q}$ & $\mathrm{N}$ & $\mathrm{R}$ & $\mathrm{R}$ & $\mathrm{M}$ & $\mathrm{K}$ & $\mathrm{W}$ & $\mathrm{K}$ & $\mathrm{K}$ & $E$ & $\mathrm{~N}$ \\
\hline En & $\mathrm{R}$ & $\mathrm{Q}$ & $\mathrm{Q}$ & $\mathrm{L}$ & $S$ & S & $\mathrm{E}$ & $\mathrm{L}$ & G & $\mathrm{L}$ & $\mathrm{N}$ & $E$ & A & Q & I & $\mathrm{K}$ & $\mathrm{I}$ & $\mathrm{W}$ & $\mathrm{F}$ & Q & $\mathrm{N}$ & $\mathrm{K}$ & $\mathrm{R}$ & A & $\mathrm{K}$ & I & $\mathrm{K}$ & $\mathrm{K}$ & $\mathrm{S}$ & \\
\hline Ftz & $\mathrm{R}$ & I & $\mathrm{D}$ & 1 & A & $\mathrm{N}$ & A & $\mathrm{L}$ & $S$ & $\mathrm{~L}$ & $S$ & $E$ & $\mathrm{R}$ & Q & I & $\mathrm{K}$ & I & $\mathrm{W}$ & $F$ & $\mathrm{Q}$ & $\mathrm{N}$ & $\mathrm{R}$ & $\mathrm{R}$ & $\mathrm{M}$ & $\mathrm{K}$ & $\mathrm{S}$ & $\mathrm{K}$ & $\mathrm{K}$ & $\mathrm{D}$ & $\mathrm{R}$ \\
\hline Mat2 & $\mathrm{L}$ & $\mathrm{E}$ & $\mathrm{N}$ & $\mathrm{L}$ & $\mathrm{M}$ & $\mathrm{K}$ & $\mathrm{N}$ & $\mathrm{T}$ & $\mathrm{S}$ & $\mathrm{L}$ & $S$ & $\mathrm{R}$ & I & Q & I & $\mathrm{K}$ & $\mathrm{N}$ & $\mathrm{W}$ & $\mathrm{V}$ & $\mathrm{S}$ & $\mathrm{N}$ & $\mathrm{R}$ & $\mathrm{R}$ & $\mathrm{R}$ & $\mathrm{K}$ & $E$ & $\mathrm{~K}$ & $\mathrm{~T}$ & I & $\mathrm{T}$ \\
\hline Vnd & $\mathrm{R}$ & $\mathrm{E}$ & $\mathrm{H}$ & $\mathrm{L}$ & A & S & $\mathrm{L}$ & I & $\mathrm{R}$ & $\mathrm{L}$ & $\mathrm{T}$ & $\mathrm{P}$ & $\mathrm{T}$ & Q & $\mathrm{V}$ & $\mathrm{K}$ & I & $\mathrm{W}$ & $\mathrm{F}$ & $\mathrm{Q}$ & $\mathrm{N}$ & $\mathrm{H}$ & $\mathrm{R}$ & $\mathrm{Y}$ & $\mathrm{K}$ & $\mathrm{T}$ & $\mathrm{K}$ & $\mathrm{R}$ & $\mathrm{A}$ & Q \\
\hline
\end{tabular}

Table S1: Sequence Alignment of Homeodomains. En stands for Engrailed, Ftz for Fushi tarazu, and Vnd for Vnd/Nk-2, * = there is a 3 residue insertion here that was taken out for alignment. Highlighted residues indicate residues conserved in all 7 homeodomains shown. 
A

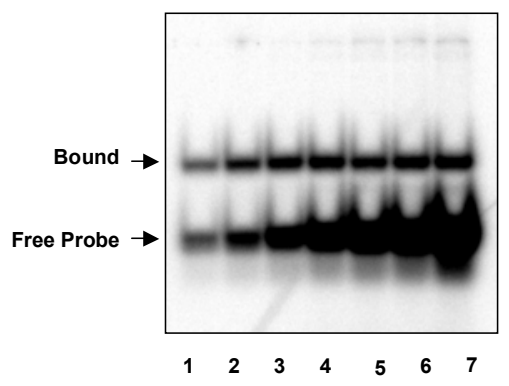

B

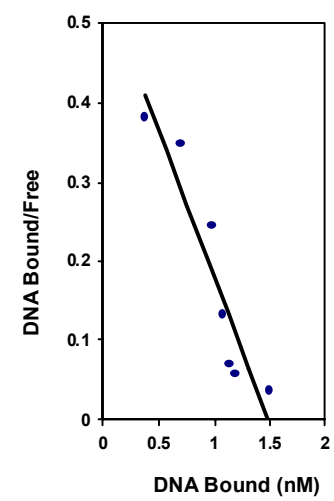

Figure S1: Gel shift assay for Scatchard analysis to determine the affinity of recombinant Pitx2 homeodomain for the bicoid consensus DNA element. (A) The DNA probe concentrations used in this analysis were 1, 2, 4, 8,16, 20 and $40 \mathrm{nM}$ for lanes 1 to 7 , respectively at a fixed protein concentration. (B) The $K D$ value obtained was $2.6 \pm 0.38 \mathrm{nM}$ (mean \pm standard deviation) from three independent DNA binding curves obtained from quantitative gel shift assays by measuring the bound and free fractions of the probes with a PhosphorImager. The data were analyzed using Microsoft Excel (linear regression analysis) to determine the $K D$ value ( $1 / K D=$ slope of the plot of Bound/Free against Bound DNA). 


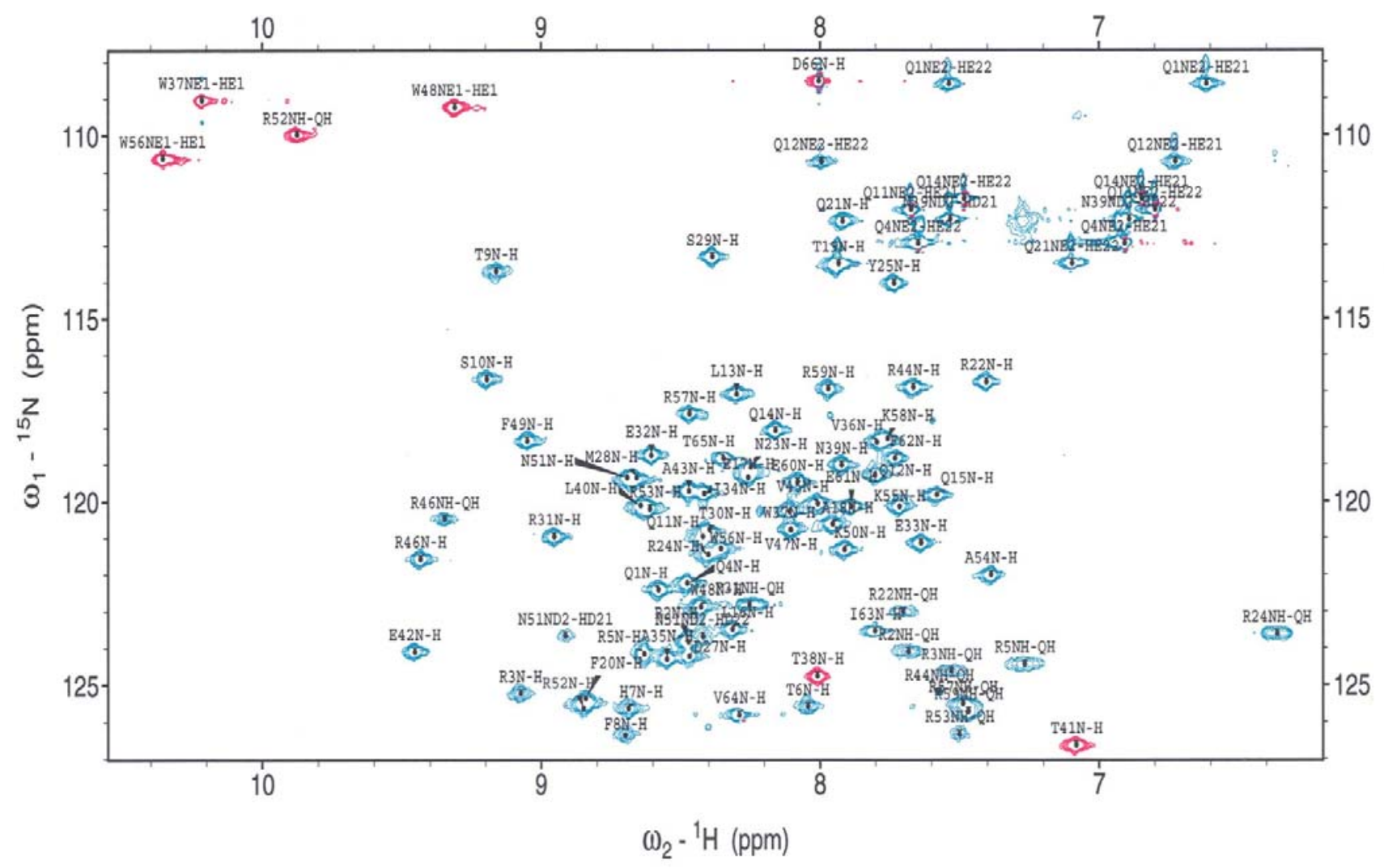

Figure S2: ${ }^{15} \mathrm{~N}-\mathrm{HSQC}$ labeled with backbone resonance and side-chain assignments obtained through triple-resonance experiments. All red peaks, and Arginine NH-QH peaks are folded in, with actual resonances listed in the following tables. 


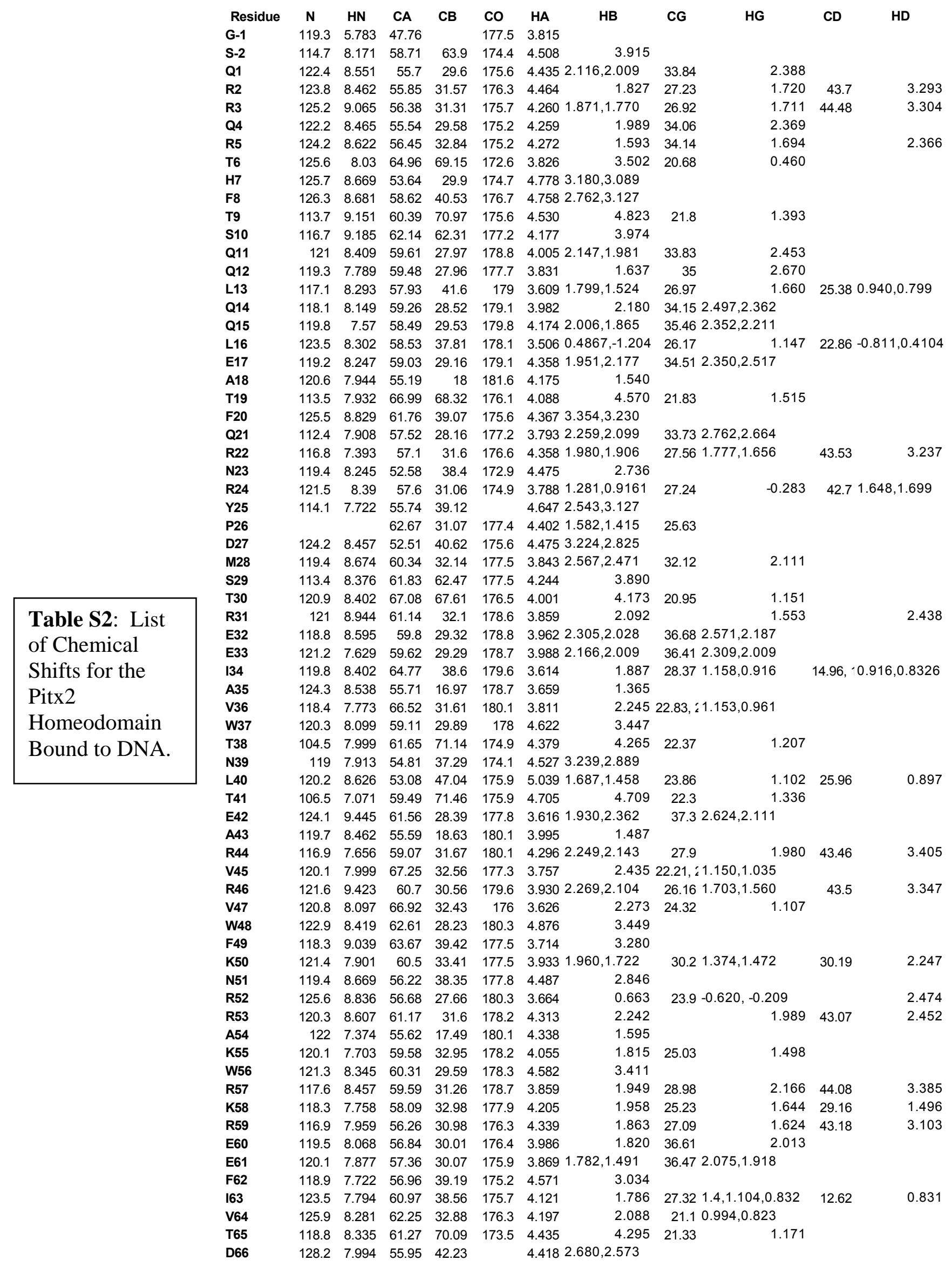




\begin{tabular}{|c|c|c|c|c|c|c|c|c|c|c|c|c|c|}
\hline Residue & NE1 & CD1/2 & HD1/2 & $\mathrm{CE} 1 / 2$ & HE1/2 & $\underline{\mathrm{CZ}}$ & $\mathrm{HZ}$ & CE3 & HE3 & $\mathrm{CZ2/3}$ & $\mathrm{HZ2/3}$ & $\underline{\mathrm{CH} 2}$ & $\mathrm{HH2}$ \\
\hline $\mathrm{H} 7$ & & 119.8 & 7.120 & 137.0 & 8.315 & & & & & & & & \\
\hline F8 & & & 7.351 & & 7.292 & & 7.075 & & & & & & \\
\hline $\mathrm{F} 20$ & & 126.7 & 7.585 & 132.2 & $7.384 / 7.44$ & 122.0 & 7.170 & & & & & & \\
\hline Y25 & & 133.6 & 7.065 & 117.8 & 6.793 & & & & & & & & \\
\hline W37 & 109.0 & 126.4 & 7.128 & & 10.210 & & & 122.0 & 7.127 & $114.7 / 120$. & $7.448 / 7.572$ & 124.7 & 7.204 \\
\hline W48 & 109.3 & 128.7 & 6.966 & & 9.301 & & & & 7.429 & 113.7 & $7.125 / 7.091$ & & 7.217 \\
\hline F49 & & 132.2 & 7.799 & & 7.769 & & 7.594 & & & & & & \\
\hline W56 & 110.6 & 127.5 & 7.473 & & 10.350 & & & 130.5 & 7.041 & $114.7 / 119$ & $7.256 / 7.694$ & 122.0 & 7.169 \\
\hline F62 & & 131.8 & 7.093 & $130.5 / 13 c$ & $7.318 / 7.318$ & & 7.295 & & & & & & \\
\hline
\end{tabular}

Table S3: Assignments of Atoms in Aromatic

Groups of the Pitx2 Homeodomain.

\begin{tabular}{crr} 
Residue & N $\boldsymbol{2}$ & \multicolumn{1}{c}{$\boldsymbol{H} \boldsymbol{}$} \\
\hline R2 & 83.8 & 7.689 \\
R3 & 84.7 & 7.478 \\
R5 & 84.5 & 7.256 \\
R22 & 83.0 & 7.652 \\
R24 & 83.6 & 6.359 \\
R31 & 83.2 & 8.186 \\
R44 & 84.6 & 7.522 \\
R46 & 80.5 & 9.330 \\
R52 & 89.9 & 9.852 \\
R53 & 86.4 & 7.495 \\
R57 & 85.6 & 7.452 \\
R59 & 85.8 & 7.396
\end{tabular}

Table S4: Chemical shift assignments of the arginine side-chains.

\begin{tabular}{crrr} 
Residue & ND2 & \multicolumn{1}{l}{ HD21 } & \multicolumn{1}{l}{ HD22 } \\
N23 & & & \\
N39 & 112.3 & 7.520 & 6.878 \\
N51 & 123.6 & 8.906 & 8.405
\end{tabular}

Table S5: Chemical shift assignments of the asparagine side-chains. 


$\begin{array}{crrrr}\text { Residue } & \text { NE2 } & \text { HE21 } & \text { HE22 } \\ \text { Q1 } & 108.6 & 6.583 & 7.514 \\ \text { Q4 } & 113.0 & 6.893 & 7.634 \\ \text { Q11 } & 112.0 & 7.663 & 6.787 \\ \text { Q12 } & 110.7 & 6.716 & 7.982 \\ \text { Q14 } & 111.7 & 6.835 & 7.472 \\ \text { Q15 } & 112.0 & 6.787 & 7.663 \\ \text { Q21 } & 113.5 & 7.910 & 7.083\end{array}$

Table S6: Chemical shift assignments of the glutamine side-chains.

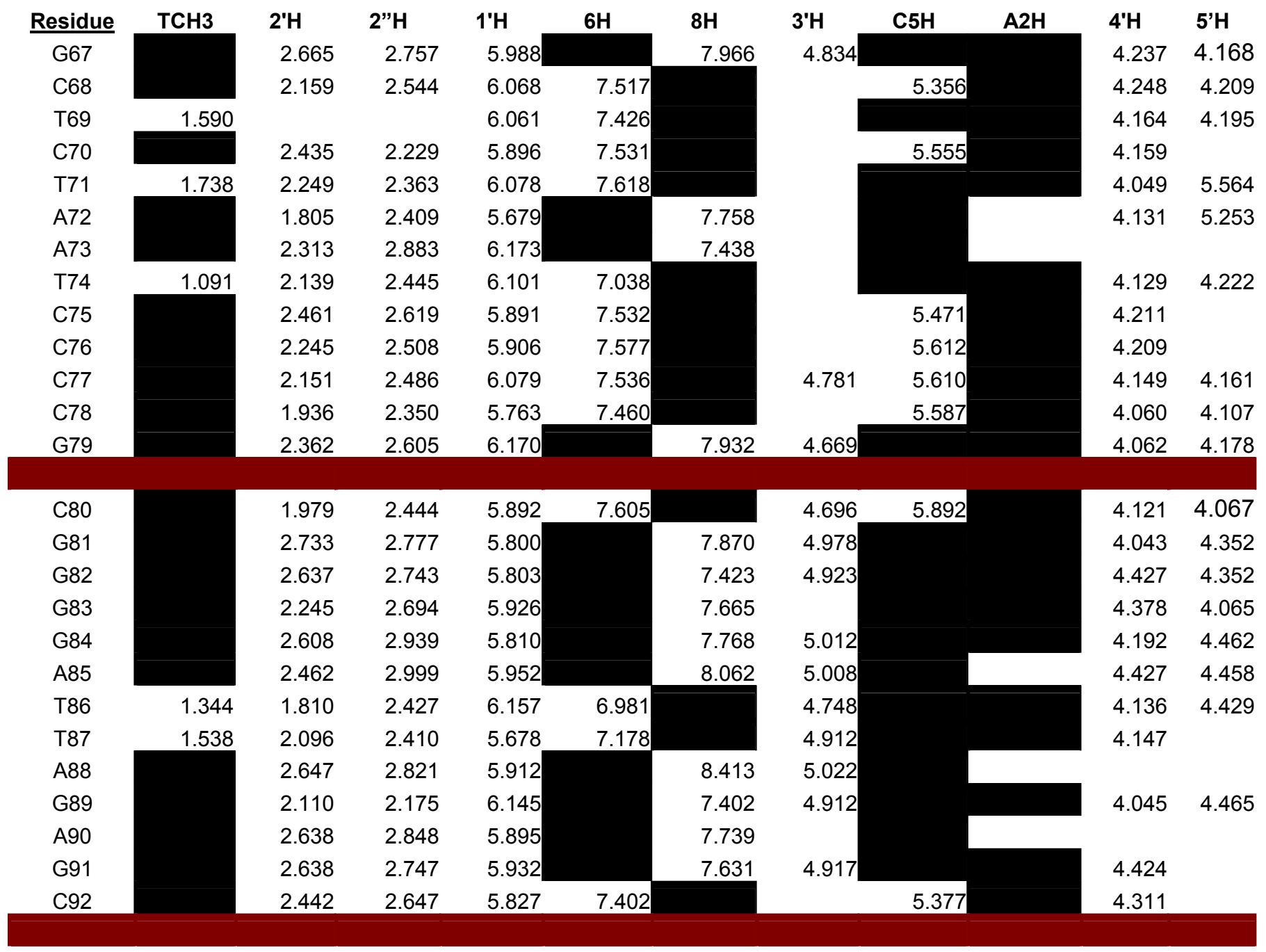

Table S7: Chemical shift assignments of the DNA binding site. 


\begin{tabular}{|c|c|c|}
\hline DNA & Protein & Upper Distance Limit ( $(\mathbf{A})$ \\
\hline T71 Q5 & R52 QB & 5.00 \\
\hline A72 4'H & R3 QD & 4.00 \\
\hline A72 Q5' & R3 Hع & 5.00 \\
\hline A72 Q5' & T6 HG & 5.00 \\
\hline A72 Q5' & V47 QG1 & 6.00 \\
\hline A72 8H & W48 HA & 6.00 \\
\hline A73 2'H & V47 QG1 & 5.00 \\
\hline A73 8H & R44 Hع & 6.00 \\
\hline A73 2"H & V47 QG1 & 5.00 \\
\hline T74 Q5' & $\mathrm{R} 2 \mathrm{HA}$ & 6.00 \\
\hline T74 Q5' & R2 QD & 6.00 \\
\hline T74 4'H & R44 QB & 6.00 \\
\hline T74 2"H & R44 QD & 6.00 \\
\hline $\mathrm{T} 746 \mathrm{H}$ & V47 QG1 & 6.00 \\
\hline T74 TCH3 & V47 QG1 & 6.00 \\
\hline G81 Q5' & Y25 HE & 6.00 \\
\hline G82 Q5' & R31 QG & 5.00 \\
\hline G82 4'H & Y25 HE & 5.00 \\
\hline G83 4'H & F49 QB & 6.00 \\
\hline G83 Q5' & F49 QB & 6.00 \\
\hline G83 4'H & R53 HG2 & 6.00 \\
\hline G83 Q5' & K50 HG2 & 6.00 \\
\hline G83 Q5' & K50 HG1 & 5.00 \\
\hline G84 8H & R46 QD & 6.00 \\
\hline G84 Q5' & K50 HG1 & 6.00 \\
\hline G89 8H & $\mathrm{R} 5 \mathrm{H} \varepsilon$ & 4.00 \\
\hline G89 8H & R5 HN & 5.00 \\
\hline
\end{tabular}

Table S8: Table of Protein-DNA NOEs 


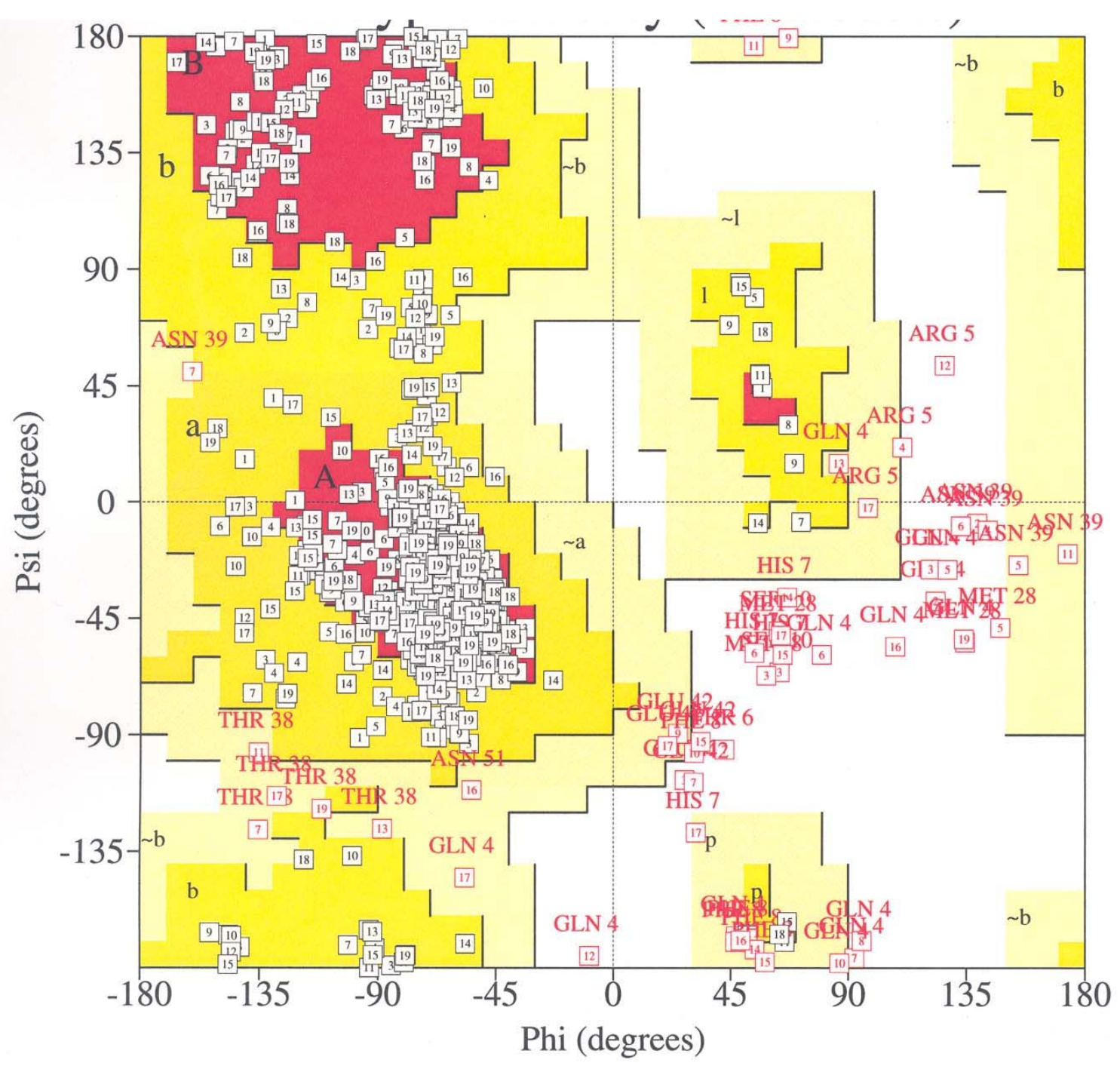

Figure S3. Ramachandran plot, generated by PROCHECK (Laskowski et al., 1993, 1996), for the 20 structures in the ensemble, for residues 3-58; $80.1 \%$ of the residues are in the most favored regions, $14.8 \%$ in the additionally allowed regions, $2.3 \%$ in generously allowed regions, and $2.8 \%$ in disallowed regions. 